\title{
Broadband (Ultraviolet to Near-Infrared) Photodetector Fabricated in n- ZnO/p-Si Nanowires Core-Shell Arrays with Ligand-Free Plasmonic Au Nanoparticles
}

\author{
$\underline{\text { Chandan Samanta }}{ }^{1}$, Sekhar Bhattacharya ${ }^{2}$, A. K. Raychaudhuri ${ }^{3}$, and Barnali Ghosh ${ }^{1 *}$ \\ ${ }^{I}$ Department of Condensed Matter Physics and Material Sciences, S. N. Bose National Centre for \\ Basic Sciences, Salt Lake, Block-JD, Sector-III, Kolkata 700106, India \\ 2Core Laboratories, King Abadullah University of Science and Technology, Saudi Arabia \\ ${ }^{3}$ CSIR-Central Glass and Ceramic Research Institute, Raja S C Mullick Road, Kolkata 700032
}

\section{Corresponding author}

* Email address: barnali@bose.res.in 


\section{Supporting figures}

The crystalline structure of the sample is identified through XRD shown in figure $\mathrm{S} 1$. The diamonds, asterisks and squares show peak belonging to Au NPs, ZnO and p-Si NWs respectively.

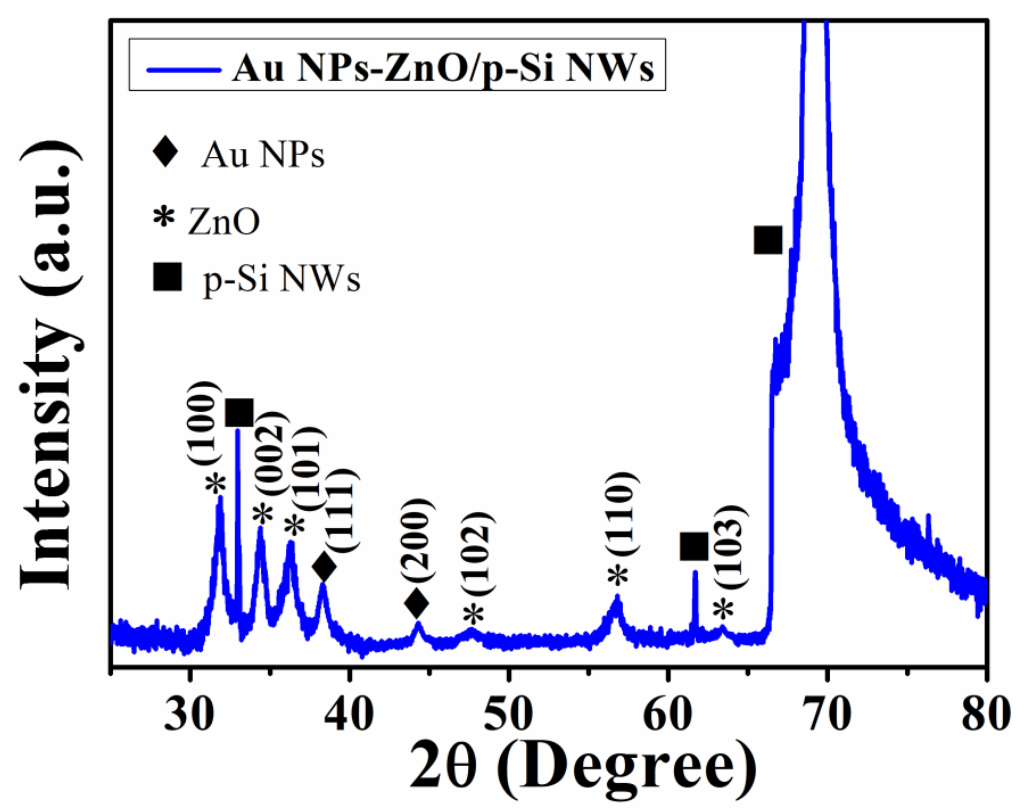

Figure S1. The XRD of Au NPs decorated ZnO/p-Si NWs heterostructure arrays.

In order to establish the physical structure of photo detector, we characterised the sample through the cross sectional TEM along with elemental analysis (EDS). Scanning tunnelling electron microscopy high angle annular dark-field (STEM-HAADF) imaging of the cross-section lamellar is shown in figure S2 (a). A composition profile of the device was obtained by EDS line scan marked by yellow arrow and the data are shown in figure S2 (b). The line scan of the $\mathrm{ZnO} / \mathrm{Si} \mathrm{NWs} \mathrm{shows} \mathrm{the} \mathrm{contents} \mathrm{of} \mathrm{Au}, \mathrm{Zn}, \mathrm{Si}$ and O. There is a clear top $\mathrm{ZnO}$ layer on the NWs array, which is followed by the conformal coating of $\mathrm{ZnO}$ through the $\mathrm{Si}$ NWs array and the concentration of Au NPs layer with thickness of about $30 \mathrm{~nm}$ is maximum on the top of $\mathrm{ZnO} / \mathrm{Si} \mathrm{NWs}$ and then concentration is uniform throughout the heterostructure arrays.

The STEM-EDS mapping for the Au M shell, Zn L shell, O K shell and Si K shell are given in Figure S3 (a) - (d) respectively. 
(a)

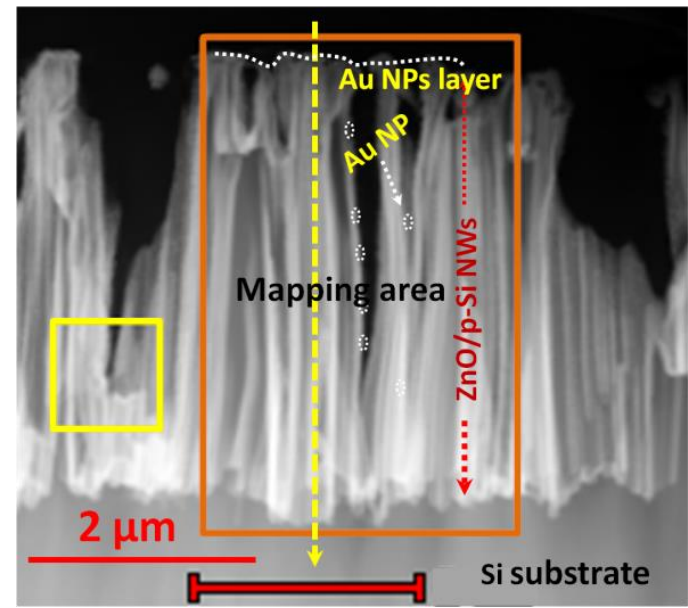

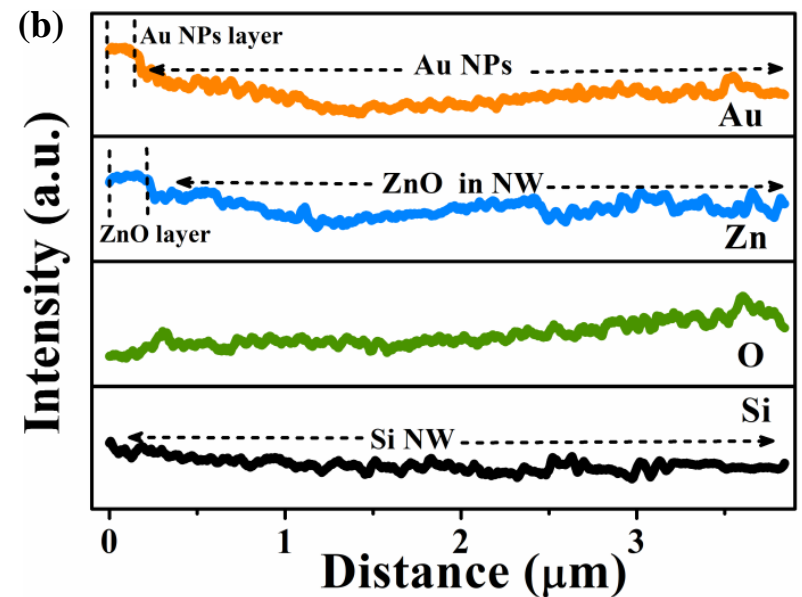

Figure S2. (a) The Cross sectional scanning tunnelling electron microscopy high angle annular dark field (STEM-HAADF) image of Au NPs decorated ZnO/Si NWs arrays. (b) Elemental composition of $\mathrm{Au}$ NPs on $\mathrm{ZnO} / \mathrm{Si}$ NWs arrays taken by EDS line scan showing the chemical composition.
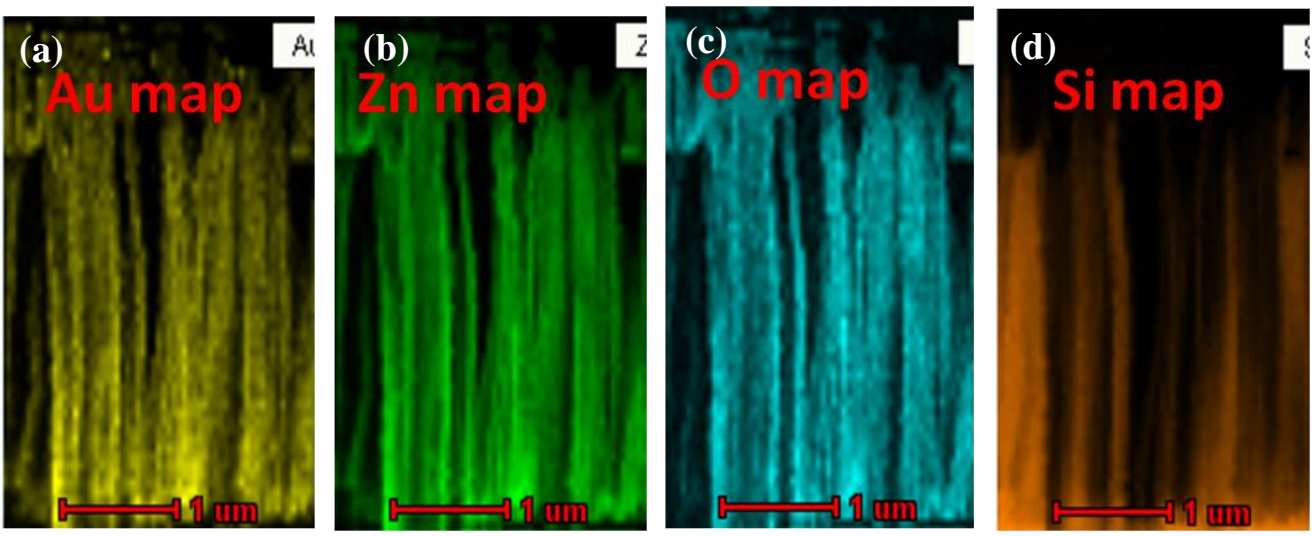

Figure S3. STEM EDS mapping of (a) Au (M shell) (b) zinc (L shell) (c) oxygen (K shell) (d) silicon (K shell) of Au NPs decorated ZnO/p-Si NWs core shell arrays.

Table S1: Comparison of device characteristics value with commercial Si photodetector

\begin{tabular}{|c|c|c|c|c|}
\hline Detectors & $\begin{array}{c}\text { Wavelength } \\
\text { range }\end{array}$ & Peak wavelength & $\begin{array}{c}\text { Responsivity } \\
(\mathbf{R})\end{array}$ & $\begin{array}{c}\text { Power } \\
\text { dissipation }\end{array}$ \\
\hline Si commercial detector & $400-1100 \mathrm{~nm}$ & $970 \mathrm{~nm}$ & $0.65 \mathrm{~A} / \mathrm{W}$ & $100 \mathrm{~mW}$ \\
\hline $\begin{array}{c}\text { Au NPs-ZnO/p-Si NWs } \\
\text { (Present work) }\end{array}$ & $300-1100$ & $800 \mathrm{~nm}$ & $1.2 \mathrm{~A} / \mathrm{W}$ & $<2 \mu \mathrm{W}$ \\
\hline
\end{tabular}




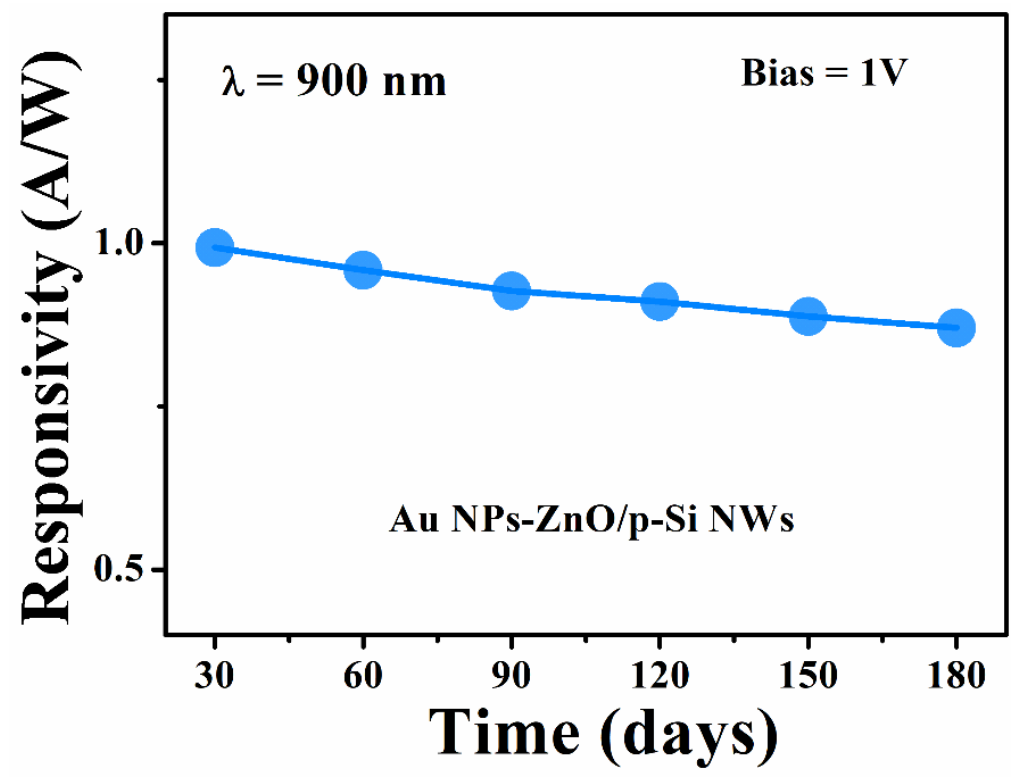

Figure S4. Stability study of photodetector during six months of time span. 